\title{
LYVE1 and PROX1 in the reconstruction of hepatic sinusoids after partial hepatectomy in mice
}

\author{
F. Meng \\ Department of Anatomy and Physiology, Shandong College of Traditional Chinese Medicine, Yantai, China \\ [Received: 27 August 2016; Accepted: 17 October 2016]
}

Background: Revascularisation is crucial to liver regeneration after liver injury, but the process remains unclear. This study investigated changes in the levels and distribution of lymphatic vessel endothelial hyaluronan receptor 1 (LYVE1) and prospero homeobox protein 1 (PROX1) in liver tissue sections after partial hepatectomy in mice.

Materials and methods: Mice were subjected to partial hepatectomy. Control animals were sham-operated. From days 1 through 8, the remaining liver tissues were collected from 8 animals each day. Histology showed that after partial hepatectomy, the remaining liver tissue samples underwent initial degeneration and then hepatocyte proliferation and regeneration. Using immunohistochemical analysis, relative to the control a significantly higher number of vascular endothelial growth factor A (VEGFA)-positive hepatocytes was observed on days 4 and 5 after partial hepatectomy.

Results: LYVE1 was mainly present in the liver sinusoidal endothelial cells and the number of LYVE1-positive cells gradually increased with time. PROX1 was detected in some of the hepatocytes, but liver sinusoidal endothelial cells, artery, and vein were negative for PROX1 staining in the early stage after liver injury. The presence of PROX1 could be observed in some central veins as well as liver sinusoidal endothelial cells. Seven days after partial hepatectomy, colocalisation of PROX1 and LYVE1 was observed in liver sinusoidal endothelial cells and veins. Conclusions: This study revealed the dynamic process of revascularisation and hepatic sinusoid reconstruction during liver regeneration in response to liver injury in mice. PROX1 and LYVE1 may participate in this process and serve as biomarkers for identification of newly formed liver sinusoidal endothelial cells. (Folia Morphol 2017; 76, 2: 239-245)

Key words: PROX1, VEGFA, LYVE1, partial hepatectomy, revascularisation

\section{INTRODUCTION}

Liver regeneration occurs in response to chronic or acute liver injury, including partial hepatectomy, with hepatocytes and epithelial cells contributing to the repair [27]. During early regeneration, the recovery of liver tissue is benefited by hepatocyte proliferation, promoted by hepatic progenitor cells $[12,28]$.
Liver sinusoidal endothelial cells, characterised by the absence of organised basement membrane and the presence of open fenestrae [7], have a pivotal role in mediating liver microcirculation during regeneration [15]. However, the mechanism of hepatic sinusoid formation and the factors that regulate this process during liver regeneration are poorly understood. 
The glycoprotein lymphatic vessel endothelial hyaluronan receptor 1 (LYVE1) is a widely accepted marker of lymphatic vessels [21] and has been observed in hepatic sinusoidal endothelial cells during the development of hepatic sinusoids [22]. Changes in the levels and distribution of LYVE1 in liver tissues during regeneration have not been fully elucidated. In our previous study, we found that after partial hepatectomy and during the reconstruction of the hepatic sinusoid, LYVE1 in the endothelial cells was induced by vascular endothelial growth factor C (VEGFC) [16].

The transcription factor prospero homeobox protein 1 (PROX1) induces the budding of lymphatic endothelial cells from a cardinal vein, and therefore is crucial to lymphangiogenesis $[5,10,31]$. In vascular endothelial cells, the presence of PROX1 has been associated with increased levels of biomarkers of lymphatic endothelial cells $[18,23]$, and in mice without PROX1 lymphatic structures were absent [31]. Nevertheless, many questions remain regarding the activity of PROX1 in liver tissues during regeneration.

The present study investigated changes in the levels and distribution of LYVE1 and PROX1 in response to liver injury. A mouse model of liver injury was created by partial hepatectomy, and the presence, levels, and distribution of LYVE1 and PROX1 was determined on days 1 through 8 after surgery.

\section{MATERIALS AND METHODS}

\section{Animals and experimental assignment}

The Experimental Animal Ethics Committee of Shandong College of Traditional Chinese Medicine approved the animal experiments. Male and female specific pathogen-free Kunming mice, weighing 18-26 g, were provided by the Experimental Animal Centre of Shandong University, China. Animals were housed at $21-23^{\circ} \mathrm{C}$ with constant humidity and a $12 \mathrm{~h}$ light/12 $\mathrm{h}$ dark lighting cycle. Mice had free access to food and water.

Before the experiments, the mice were randomly apportioned to either a sham-operated control group, or a group subjected to partial hepatectomy (HPX group).

\section{Mouse partial hepatectomy model}

To generate a mouse model of liver injury by partial hepatectomy, mice were anesthetised with $50 \mathrm{mg} / \mathrm{kg}$ ketamine and $40 \mathrm{mg} / \mathrm{kg}$ xylazine (free base) by intraperitoneal injection. A 3-cm midline incision was made in the upper abdomen. The left lateral lobe of the liver was exposed, and approximately $0.2 \mathrm{~g}$ of liver tissue was resected. The incision was then closed with 4-0 suture, and the area sterilised with ethanol. In the sham-operated (control) group the procedure was identical, except that the hepatectomy was not performed. After surgery, the mice were returned to a $37^{\circ} \mathrm{C}$ incubator to recover.

\section{Haematoxylin and eosin staining}

On days 1 to 8 after the surgery, 8 mice from each group were killed. The left lateral lobe of the liver was removed and fixed in $40 \mathrm{~g} / \mathrm{L}$ formaldehyde. After the tissues were embedded in paraffin, they were sectioned into $4-\mu \mathrm{m}$ thick slices. For histological analysis, samples were stained with haematoxylin and eosin. Images were captured under a DP-72 Olympus microscope (Japan) at a magnification of $400 \times$.

\section{Immunohistochemical analysis and imaging analysis}

The presence of PROX1, LYVE1, and vascular endothelial growth factor A (VEGFA) were examined using immunohistochemistry. In brief, paraffin-embedded tissue samples were deparaffinised. Antigen retrieval was performed by incubating samples in $0.01 \mathrm{M}$ citrate buffer for $15 \mathrm{~min}$ at $121^{\circ} \mathrm{C}$. Endogenous peroxidase was inactivated by incubating samples in $3 \mathrm{~mL} / \mathrm{L}$ hydrogen peroxide. After blocking, samples were stained serially with rabbit anti-mouse antiPROX1 antibody (1:200 dilution, Boster Biological Technology, Wuhan, China), goat anti-mouse antiLYVE1 antibody (1:100 dilution, R\&D Systems), or rabbit anti-mouse anti-VEGFA antibody (1:100 dilution, Boster Biological Technology, Wuhan, China) at $4^{\circ} \mathrm{C}$ overnight. After washing, samples were probed with horseradish peroxidase (HRP)-conjugated goat anti-rabbit or rabbit anti-goat lgG secondary antibody (Boster Biological Technology, Wuhan, China).

Immunoreactivities were visualised using a 3, 3'-diaminobenzidine tetrahydrochloride (DAB) kit in accordance with the manufacturer's instructions (Boster Biological Technology, Wuhan, China). Images were captured under a DP-72 Olympus microscope (Japan) at a magnification of $400 \times$. The mean optical density of 50 pixels was measured by Image-Pro Plus 6.0 software (Media Cybernetics, USA).

For the immunofluorescence study, samples were incubated with rabbit anti-mouse anti-PROX1 antibody (1:100 dilution) and goat anti-mouse anti-LYVE1 antibody (1:50 dilution) at $4^{\circ} \mathrm{C}$ overnight. After washing, samples were incubated with Cy3-labeled donkey anti- 
a

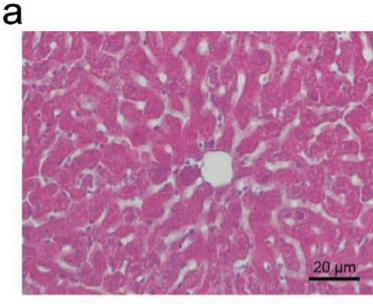

C

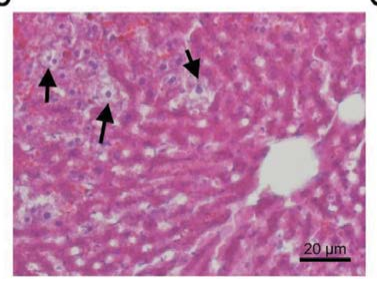

Figure 1. Haematoxylin and eosin staining of remaining liver tissue sections after partial hepatectomy; a. Control sample on day 1 after the sham operation. Hepatectomy tissue sample on day 1 (b), day 4 (c), and day 7 (d) after partial hepatectomy. Arrows in panel b indicate hepatocytes with swelling and vacuolation. Arrows in panels $\mathbf{c}$ and $\mathbf{d}$ indicate enlarged and proliferating hepatocytes; $n=8$ for each group. Original magnification: $400 \times$. Scale bar: $20 \mu \mathrm{m}$.

goat lgG or fluorescein isothiocyanate (FITC)-labelled donkey anti-rabbit IgG antibodies (Antgene). Immunofluorescence was examined under a DP-72 fluorescence microscope (Olympus, Japan) at a magnification of 200x. The number of PROX1- and LYVE1-positive veins within 50 pixels of each slice was counted.

\section{Statistical analysis}

Data were analysed using Microsoft Excel software. Comparisons between groups were performed using Student's $t$-test. $\mathrm{P}<0.05$ was considered a significant difference.

\section{RESULTS}

\section{Histological changes}

The daily food and water consumption of the control (sham operated) group was $3.47 \pm 0.47 \mathrm{~g} / \mathrm{d}$ and $4.13 \pm 0.71 \mathrm{~mL} / \mathrm{d}$, respectively, and in the HPX group was $6.05 \pm 0.84 \mathrm{~g} / \mathrm{d}$ and $6.15 \pm 0.73 \mathrm{~mL} / \mathrm{d}$. On postoperative day 8 , the average body weight gain in the control and HPX groups was $0.20 \pm 0.00 \mathrm{~g} / \mathrm{kg}$ and $0.17 \pm 0.05 \mathrm{~g} / \mathrm{kg}$. The mortality rate was $1 \%$ in the control and $30 \%$ in the HPX group.

In the control group, 1 day after the surgery, the structure of the remaining liver tissue section was intact (Fig. 1). No histological differences were observed in the liver tissue sections of the control group between days 1 and 8 post-surgery (data not shown).
In the HPX group, 1 day after the partial hepatectomy, the following obvious histological changes in the remaining liver tissue section were detected (Fig. 1b): disordered structure of the hepatic lobule; swelling, and vacuolation in hepatocytes; red blood cell accumulation in the obscure hepatic sinusoid; and loss of typical hepatic cord organisation. Four days after the surgery enlarged hepatocytes and cells containing dual nuclei could be observed (Fig. 1c), suggesting that these cells were under proliferation. Red blood cell accumulation, disorganised hepatic sinusoids and hepatic cords were still visible. Seven days after the surgery, an improvement in the structure of the hepatic lobule was found. However, enlarged hepatocytes, surrounded by red blood cells, could still be detected (Fig. 1d). Therefore, it appears that after the partial hepatectomy, the remaining liver tissue samples underwent initial degeneration, and then regeneration.

\section{Presence of VEGFA in liver tissue sections}

VEGFA was mainly observed in the cytoplasm of the cells. In the control group, the amount of VEGFA was low relative to that of the HPX group, and only a small number of VEGFA-positive cells could be detected surrounding the central vein (Fig. 2A), and VEGFA staining on postoperative days 1 and 7 was similar ( $p>0.05$; Fig. 2A, B).

In the HPX group, there was a significant increase in the number of VEGFA-positive hepatocytes on postoperative days 4 and 5 .

\section{Presence of LYVE1 in liver tissue section}

To understand the involvement of LYVE1 in hepatic pathogenesis and liver regeneration after partial hepatectomy, the presence of LYVE1 was investigated using immunohistochemistry (Fig. 3A). In the control group, LYVE1 was present mainly on the membranes of some sinusoids of the hepatic lobule, and absent from the arteries and veins, including the central veins.

In the HPX group, one day after the surgery there was deformation of the LYVE1-positive hepatic sinusoid, caused by enlarged hepatocytes. Although the structure of the hepatic sinusoid was recovered at 4 days after the surgery, no LYVE1-positive central vein was found. On postoperative day 7, the number of LYVE1-positive hepatic sinusoids was significantly increased. The semiquantitative analysis revealed that the LYVE1 staining intensity was significantly elevated since day 3 after surgery compared with other time-points (Fig. 3B). 
A

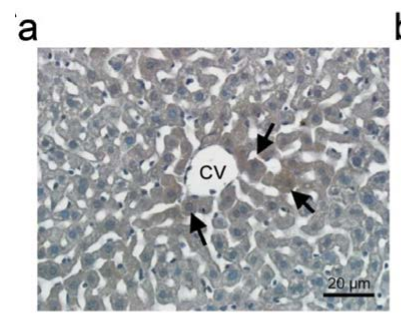

C

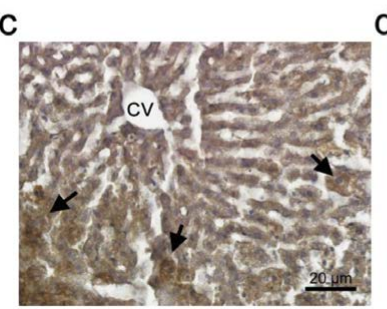

b

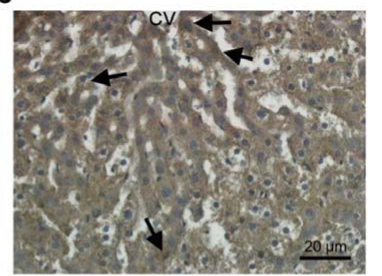

d

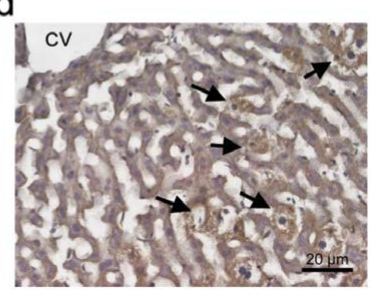

B

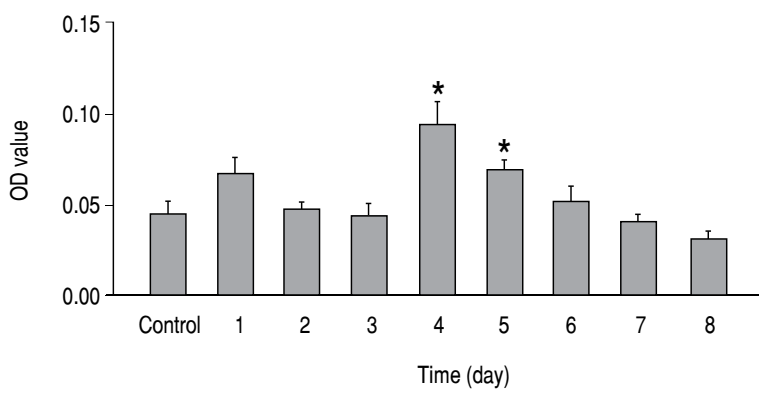

Figure 2. Immunohistochemical analysis of VEGFA in remaining liver tissue sections after partial hepatectomy; A. Samples derived from liver tissues of (a) control mice on day 1 after the sham operation, and in hepatectomy mice on day 1 (b), day $4(\mathbf{c})$, and day 7 (d) after partial hepatectomy. Arrows indicate VEGFA-positive hepatocytes; $\mathrm{CV}$ - central vein; $\mathrm{n}=8$ for each group. Original magnification: $400 \times$. Scale bar: $20 \mu \mathrm{m}$; B. Mean optical density (OD) of VEGFA staining; ${ }^{*} p<0.05$ compared with control.

\section{Presence of PROX1 in liver tissue sections}

In the control group, the amount of PROX1 in the hepatic lobule of control mice was low but uniformly distributed in the cytoplasm of hepatocytes (Fig. 4), and the liver sinusoidal endothelial cells were negative for PROX1 staining.

In the HPX group, 1 day after surgery the number of PROX1-positive hepatocytes was significantly less than that of the control. On day 4, the cytoplasmic staining of PROX1 was detected in hepatocytes, and no PROX1-positive hepatic sinusoid could be observed. On day 7 , the number of PROX1-positive hepatocytes was greatly increased. In addition, the presence of PROX1 could be observed in some central veins as well as liver sinusoidal endothelial cells.
A

a

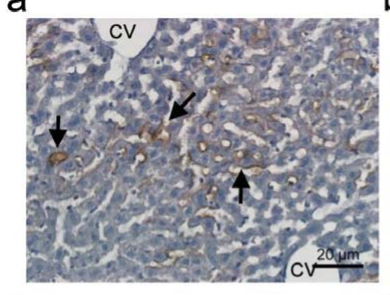

b

C

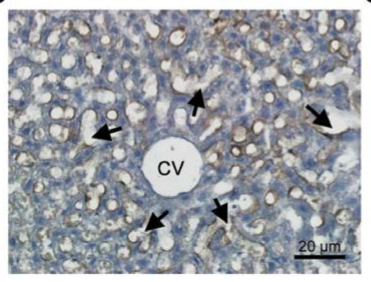

d
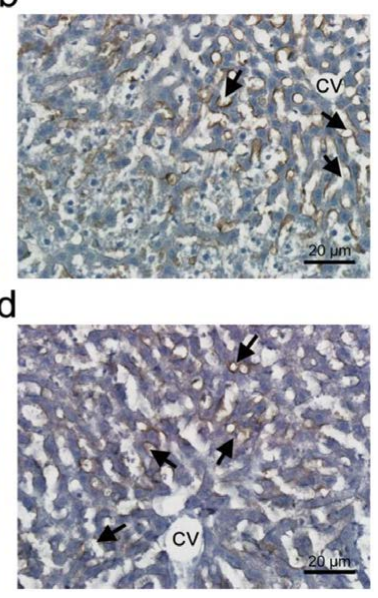

B

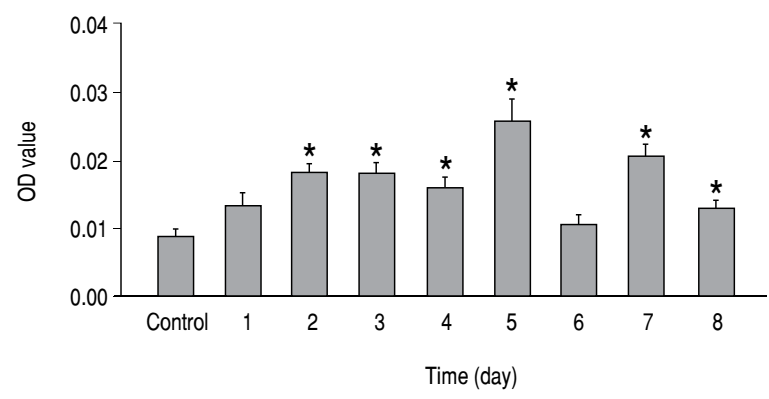

Figure 3. Immunohistochemical analysis of LYVE1 in remaining liver tissue sections after partial hepatectomy; A. Samples derived from liver tissues of (a) control mice on day 1 after the sham operation, and in hepatectomy mice on day 1 (b), day 4 (c), and day 7 (d) after partial hepatectomy. Arrows indicate the LVVE1-positive vascular endothelial cells; $\mathrm{CV}$ - central vein; $\mathrm{n}=8$ for each group. Original magnification: $400 \times$. Scale bar: $20 \mu \mathrm{m}$; B. Mean optical density (OD) of LYVE1 staining; ${ }^{*} p<0.05$ compared with control.

\section{Colocalisation of LYVE1 and PROX1 in vein and liver sinusoidal endothelial cells}

To understand the association between LYVE1 and PROX1 in liver regeneration, immunostaining with both anti-LYVE1 and anti-PROX1 antibodies was performed (Fig. 5). In the HPX group, on day 7 after the surgery there was a significant colocalisation of LYVE1 and PROX1 in veins and in liver sinusoidal endothelial cells. The semi-quantitative analysis showed that the number of LYVE1- and PROX1-positive veins was significantly elevated from day 5 to day 7 after partial hepatectomy compared with other time points.

\section{DISCUSSION}

Liver regeneration is a complex process with a variety of cells, factors, and signalling pathways involved [17]. 
A

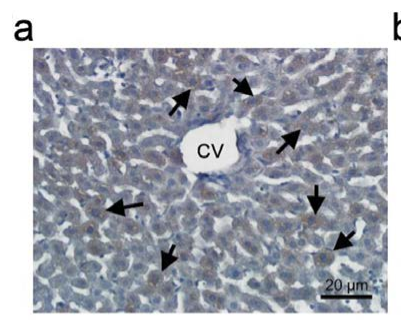

C

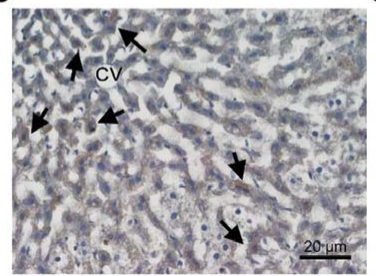

B

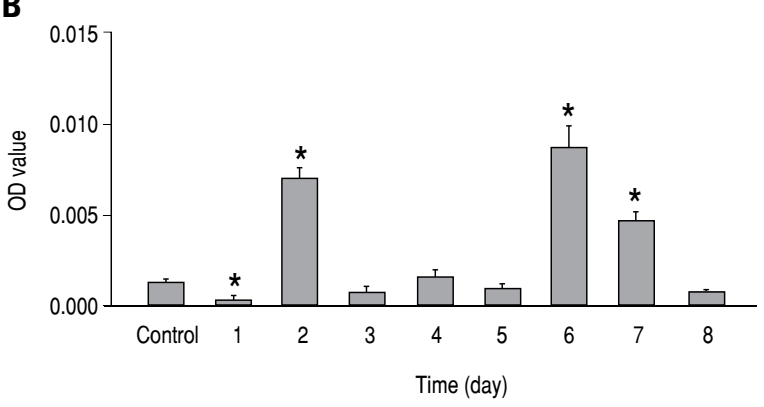

Figure 4. Immunohistochemical analysis of PROX1 in remaining liver tissue sections after partial hepatectomy; A. Samples derived from liver tissues of (a) control mice on day 1 after the sham operation, and in hepatectomy mice on day 1 (b), day 4 (c), and day 7 (d) after partial hepatectomy. Arrows indicate the PROX1-positive vascular endothelial cells; CV - central vein; $n=8$ for each group. Original magnification: $400 \times$. Scale bar: $20 \mu \mathrm{m}$; B. The mean optical density (OD) of PROX1 staining was measured; ${ }^{*} p<0.05$ compared with control.

Liver sinusoidal endothelial cells have an essential role during liver regeneration, as these cells, originating from bone marrow, have been found to promote hepatocyte proliferation and accelerate liver mass repair in response to injury [30]. However, the molecular mechanisms involved in these processes remain largely unknown. In this present study, we report the involvement of LYVE1 and PROX1 in the reconstruction of hepatic sinusoid after partial hepatectomy.

Partial hepatectomy, a surgical resection in the liver, has been widely adopted to induce liver injury in rodents with advantages such as moderate injury, low death rate, and rapid recovery [20]. This animal model mimics early acute liver degeneration, and then hepatocyte proliferation and hepatic vascular reconstruction during a regeneration phase in patients after hepatic resection [1].
A
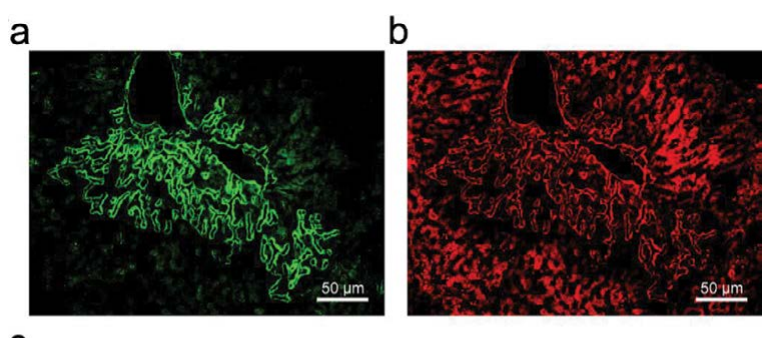

C

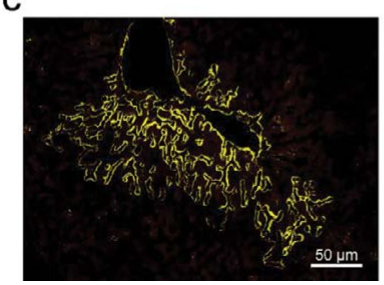

B

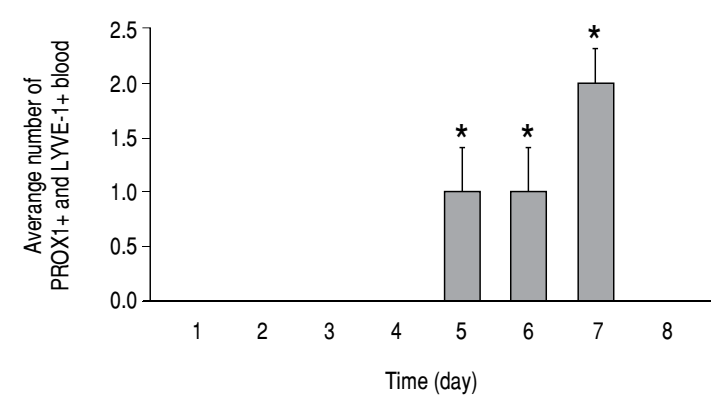

Figure 5. Immunostaining analysis of the colocalisation of LYVE1 and PROX1 in vein and liver sinusoidal endothelial cells; A. Liver tissue samples derived from animals 7 days after partial hepatectomy were stained with anti-LYVE1 (green, a) and anti-PROX1 (red, b) antibodies, and the immunoreactivities were examined under a fluorescence microscope; a merged micrograph (yellow, c); $n=8$ for each group. Original magnification: $200 \times$. Scale bar: $50 \mu \mathrm{m}$; B. Average number of LYVE1- and PROX1-positive veins; ${ }^{*} p<0.05$ compared with control.

Upon early liver regeneration, VEGF has a key role in mediating angiogenetic gene expression and modulating microvasculature [6]. In addition, VEGF regulates the recruitment of rat liver progenitor sinusoidal endothelial cells to the liver, which is a crucial step for regeneration [29]. VEGF is a large family of 5 members in mammals, namely VEGFA, VEGFB, VEGFC, VEGFD, and placental growth factor. The regulatory effect of VEGFA regarding vasculogenesis has led to its extensive study. Thus, VEGFA is generally abbreviated as VEGF [14]. In the present study, an evident increase in VEGFA levels was detected on postoperative days 4 and 5 , providing a clue to the initiation of liver regeneration. In accord with our finding, Kraizer et al. [13] reported a dynamic fluctuation of VEGF after partial hepatectomy, as the mRNA expression of VEGF increased; subsequently these 
levels gradually declined to baseline after the operation. Similar results were obtained in a rat model of partial hepatectomy [25]. The intra-platelet VEGFA level was elevated during liver regeneration in hepatocellular carcinoma patients who underwent partial hepatectomy [2]. In a rat study, the exogenous administration of VEGF at the early stage after partial hepatectomy improved liver regeneration [3]. These data suggest that VEGFA may have an essential role in mediating vasculogenesis during the early stage of liver regeneration.

LYVE1 is a lymphatic vessel marker that is mainly present in the lymphatic endothelial cells surrounding great vessels in the liver tissues [4, 21]. LYVE1 has also been detected in normal hepatic blood sinusoidal endothelial cells [19]. However, an immunohistochemical analysis showed that LYVE1 is absent from the arterial and venular endothelium, both in mouse and human adult liver [19]. In accordance with these observations, in the present study we detected LYVE1 in some of the hepatic sinusoids in mice of the control (sham-operated) group. The LYVE1 staining intensity was significantly elevated since day 3 after surgery when compared to other time points and the number of LYVE1-positive hepatic sinusoids was increased in accompanied with the time after partial hepatectomy. Seven days after surgery, LVVE1 was present in the central vein. During the development of the hepatic vascular system, LYVE1 was prominent in most of the vascular endothelial cells in liver tissues of mice at 1 day after birth; LYVE1 was found only in some of the hepatic sinusoids, but was absent from vascular endothelial cells $[9,22]$. Together with our findings, these data suggest that LYVE1 levels in the liver and its distribution changes during development and in response to injury.

PROX1, mainly present in hepatic progenitors [24], has an important role in the regulation of hepatocyte proliferation and migration [11, 26]. However, PROX1 is absent from hepatic sinusoids or venous endothelia due to the specification of progenitor cells [8]. In our present study, nuclear accumulation of PROX1 was detected in hepatocytes after partial hepatectomy. This suggests that PROX1-mediated hepatocyte regeneration occurs in response to liver injury. In addition, the presence of PROX1 was also detected in some central veins, as well as in the surrounding liver sinusoidal endothelial cells, indicating the involvement of PROX1 in hepatic sinusoid reconstruction after liver injury. Collectively, these data suggest that PROX1 may participate in hepatocyte proliferation and hepatic sinusoid reconstruction during liver regeneration.
Although there are several biomarkers specific to liver sinusoidal endothelial cells, they share similarities with lymphatic endothelial cells in function and phenotype [22]. PROX1 has been found to drive the budding of lymphatic endothelial cells from the cardinal vein and induce differentiation from venous vascular endothelial cells into LYVE1-positive lymphatic endothelial cells $[5,10,31]$. In the present study, the number of LYVE1- and PROX1-positive vessels gradually increased over time after liver injury. However, the blood vessels that were positive for both LYVE1 and PROX1 were absent on day 8. Therefore, it is possible that newly formed hepatic sinusoids and venous endothelial cells contain both LYVE1 and PROX1, and PROX1 may induce the differentiation of LYVE1-positive liver sinusoidal endothelial cells. These results suggest that LYVE1 and PROX1 are involved in revascularisation after liver injury.

\section{CONCLUSIONS}

In summary, our current findings show that the levels and distribution of LYVE1 and PROX1 are dynamic during the reconstruction of hepatic sinusoids after partial hepatectomy in mice. However, this study also has some limitations, as the sequential production of these proteins during hepatic regeneration has not been fully determined, and the protein levels need to be quantified. Our results provide valuable evidence for understanding revascularisation and hepatic sinusoid reconstruction during liver regeneration in response to liver injury, and suggest that LYVE1 and PROX1 may serve as biomarkers for newly formed liver sinusoidal endothelial cells.

\section{Acknowledgements}

This study was supported by Administration of Traditional Chinese Medicine of the Shandong Province (Grant No. 2013-149).

\section{REFERENCES}

1. Abbas $M$, Farouk $Y$, Hassan $A A$, et al. Perioperative acute phase response and outcome of hepatic resection, an experimental study. J Egypt Soc Parasitol. 2013; 43(2): 341-350, indexed in Pubmed: 24260813.

2. Aryal B, Shimizu T, Kadono J, et al. A switch in the dynamics of intra-platelet VEGF-A from cancer to the later phase of liver regeneration after partial hepatectomy in humans. PLoS One. 2016; 11(3): e0150446, doi: 10.1371/journal. pone.0150446, indexed in Pubmed: 26930285.

3. Assy N, Spira G, Paizi M, et al. Effect of vascular endothelial growth factor on hepatic regenerative activity following partial hepatectomy in rats. J Hepatol. 1999; 30(5): 911-915, indexed in Pubmed: 10365820. 
4. Banerji S, Ni J, Wang SX, et al. LYVE-1, a new homologue of the CD44 glycoprotein, is a lymph-specific receptor for hyaluronan. J Cell Biol. 1999; 144(4): 789-801, indexed in Pubmed: 10037799.

5. Betterman KL, Harvey NL. The lymphatic vasculature: development and role in shaping immunity. Immunol Rev. 2016; 271(1): 276-292, doi: 10.1111/imr.12413, indexed in Pubmed: 27088921.

6. Bockhorn M, Goralski M, Prokofiev D, et al. VEGF is important for early liver regeneration after partial hepatectomy. J Surg Res. 2007; 138(2): 291-299, doi: 10.1016/j. jss.2006.07.027, indexed in Pubmed: 17275844.

7. DeLeve LD, Jaeschke $H$, Kalra VK, et al. 15th International Symposium on Cells of the Hepatic Sinusoid, 2010. Liver Int. 2011; 31(6): 762-772, doi: 10.1111/j.14783231.2011.02527.x, indexed in Pubmed: 21645207.

8. Dudas J, Papoutsi M, Hecht M, et al. The homeobox transcription factor Prox 1 is highly conserved in embryonic hepatoblasts and in adult and transformed hepatocytes, but is absent from bile duct epithelium. Anat Embryol (Berl). 2004; 208(5): 359-366, doi: 10.1007/s00429-0040403-4, indexed in Pubmed: 15232737.

9. Gordon EJ, Gale NW, Harvey NL. Expression of the hyaluronan receptor LYVE-1 is not restricted to the lymphatic vasculature; LYVE-1 is also expressed on embryonic blood vessels. Dev Dyn. 2008; 237(7): 1901-1909, doi: 10.1002/ dvdy.21605, indexed in Pubmed: 18570254.

10. Hong YK, Harvey N, Noh YH, et al. Prox 1 is a master control gene in the program specifying lymphatic endothelial cell fate. Dev Dyn. 2002; 225(3): 351-357, doi: 10.1002/ dvdy.10163, indexed in Pubmed: 12412020.

11. Kamiya A, Kakinuma S, Onodera M, et al. Prospero-related homeobox 1 and liver receptor homolog 1 coordinately regulate long-term proliferation of murine fetal hepatoblasts. Hepatology. 2008; 48(1): 252-264, doi: 10.1002/ hep.22303, indexed in Pubmed: 18571787.

12. Kitade M, Kaji K, Yoshiji H. Relationship between hepatic progenitor cell-mediated liver regeneration and non-parenchymal cells. Hepatol Res. 2016; 46(12): 1187-1193, doi: 10.1111/hepr.12682, indexed in Pubmed: 26895456.

13. Kraizer $\mathrm{Y}$, Mawasi N, Seagal J, et al. Vascular endothelial growth factor and angiopoietin in liver regeneration. Biochem Biophys Res Commun. 2001; 287(1): 209-215, doi: 10.1006/bbrc.2001.5548, indexed in Pubmed: 11549276.

14. Licht T, Keshet E. Delineating multiple functions of VEGF$A$ in the adult brain. Cell Mol Life Sci. 2013; 70(10): 1727-1737, doi: 10.1007/s00018-013-1280-x, indexed in Pubmed: 23475068.

15. Marrone G, Shah VH, Gracia-Sancho J. Sinusoidal communication in liver fibrosis and regeneration. J Hepatol. 2016; 65(3): 608-617, doi: 10.1016/j.jhep.2016.04.018, indexed in Pubmed: 27151183.

16. Meng F. [Vascular endothelial growth factor $C$ induces LYVE-1(+) endothelial cells to reconstruct hepatic sinusoid during liver regeneration]. Xi Bao Yu Fen Zi Mian Yi Xue Za Zhi. 2014; 30(10): 1039-1042, indexed in Pubmed: 25270205.

17. Michalopoulos GK. Liver regeneration after partial hepatectomy: critical analysis of mechanistic dilemmas. Am J Pathol. 2010; 176(1): 2-13, doi: 10.2353/ ajpath.2010.090675, indexed in Pubmed: 20019184.
18. Mishima K, Watabe T, Saito A, et al. Prox1 induces lymphatic endothelial differentiation via integrin alpha9 and other signaling cascades. Mol Biol Cell. 2007; 18(4): 1421-1429, doi: 10.1091/mbc.E06-09-0780, indexed in Pubmed: 17287396.

19. Mouta Carreira C, Nasser SM, di Tomaso E, et al. LYVE-1 is not restricted to the lymph vessels: expression in normal liver blood sinusoids and down-regulation in human liver cancer and cirrhosis. Cancer Res. 2001; 61(22): 8079-8084, indexed in Pubmed: 11719431.

20. Nikfarjam M, Malcontenti-Wilson $C$, Fanartzis $M$, et al. A model of partial hepatectomy in mice. J Invest Surg. 2004; 17(5): 291-294, doi: 10.1080/08941930490502871, indexed in Pubmed: 15385262.

21. Nishida-Fukuda $H$, Araki $R$, Shudou $M$, et al. Ectodomain Shedding of Lymphatic Vessel Endothelial Hyaluronan Receptor 1 (LYVE-1) Is Induced by Vascular Endothelial Growth Factor A (VEGF-A). J Biol Chem. 2016; 291(20): 10490-10500, doi: 10.1074/jbc.M115.683201, indexed in Pubmed: 26966180.

22. Nonaka H, Tanaka M, Suzuki K, et al. Development of murine hepatic sinusoidal endothelial cells characterized by the expression of hyaluronan receptors. Dev Dyn. 2007; 236(8): 2258-2267, doi: 10.1002/dvdy.21227, indexed in Pubmed: 17626278.

23. Petrova TV, Mäkinen T, Mäkelä TP, et al. Lymphatic endothelial reprogramming of vascular endothelial cells by the Prox-1 homeobox transcription factor. EMBO J. 2002; 21(17): 4593-4599, indexed in Pubmed: 12198161.

24. Seth A, Ye J, Yu N, et al. Prox1 ablation in hepatic progenitors causes defective hepatocyte specification and increases biliary cell commitment. Development. 2014; 141(3): 538-547, doi: 10.1242/dev.099481, indexed in Pubmed: 24449835.

25. Shimizu H, Mitsuhashi N, Ohtsuka M, et al. Vascular endothelial growth factor and angiopoietins regulate sinusoidal regeneration and remodeling after partial hepatectomy in rats. World J Gastroenterol. 2005; 11(46): 7254-7260, indexed in Pubmed: 16437624.

26. Sosa-Pineda B, Wigle JT, Oliver G. Hepatocyte migration during liver development requires Prox1. Nat Genet. 2000; 25(3): 254-255, doi: 10.1038/76996, indexed in Pubmed: 10888866.

27. Stanger BZ. Cellular homeostasis and repair in the mammalian liver. Annu Rev Physiol. 2015; 77: 179-200, doi: 10.1146/annurev-physiol-021113-170255, indexed in Pubmed: 25668020.

28. Tu T, Calabro SR, Lee A, et al. Hepatocytes in liver injury: Victim, bystander, or accomplice in progressive fibrosis? J Gastroenterol Hepatol. 2015; 30(12): 1696-1704, doi: 10.1111/jgh.13065, indexed in Pubmed: 26239824.

29. Wang L, Wang X, Wang L, et al. Hepatic vascular endothelial growth factor regulates recruitment of rat liver sinusoidal endothelial cell progenitor cells. Gastroenterology. 2012; 143(6): 1555-1563.e2, doi: 10.1053/j. gastro.2012.08.008, indexed in Pubmed: 22902870.

30. Wang L, Wang X, Xie G, et al. Liver sinusoidal endothelial cell progenitor cells promote liver regeneration in rats. J Clin Invest. 2012; 122(4): 1567-1573, doi: 10.1172/ JCI58789, indexed in Pubmed: 22406533.

31. Wigle JT, Harvey N, Detmar M, et al. An essential role for Prox 1 in the induction of the lymphatic endothelial cell phenotype. EMBO J. 2002; 21(7): 1505-1513, doi: 10.1093/ emboj/21.7.1505, indexed in Pubmed: 11927535. 